\title{
Performance evaluation of simple linear iterative clustering algorithm on medical image processing
}

\author{
Jinyu Cong ${ }^{\mathrm{a}}$, Benzheng Wei ${ }^{\mathrm{a},}$, Yilong Yin ${ }^{\mathrm{b}}$, Xiaoming $\mathrm{Xi}^{\mathrm{b}}$ and Yuanjie Zheng ${ }^{\mathrm{c}}$ \\ ${ }^{a}$ College of Science and Technology, Shandong University of Traditional Chinese Medicine, Jinan \\ 250355, China \\ ${ }^{b}$ School of Computer Science and Technology, Shandong University, Jinan 250100, China \\ ${ }^{c}$ School of Medicine, University of Pennsylvania, Philadelphia 19104, USA
}

\begin{abstract}
Simple Linear Iterative Clustering (SLIC) algorithm is increasingly applied to different kinds of image processing because of its excellent perceptually meaningful characteristics. In order to better meet the needs of medical image processing and provide technical reference for SLIC on the application of medical image segmentation, two indicators of boundary accuracy and superpixel uniformity are introduced with other indicators to systematically analyze the performance of SLIC algorithm, compared with Normalized cuts and Turbopixels algorithm. The extensive experimental results show that SLIC is faster and less sensitive to the image type and the setting superpixel number than other similar algorithms such as Turbopixels and Normalized cuts algorithms. And it also has a great benefit to the boundary recall, the robustness of fuzzy boundary, the setting superpixel size and the segmentation performance on medical image segmentation.
\end{abstract}

Keywords: Medical image, superpixels, SLIC, image segmentation, performance evaluation

\section{Introduction}

As a principal technique of the medical image processing and clinical application, medical image segmentation refers to partitioning an image into several disjoint subregions according to its different characteristics and meanings [1]. Unlike natural image segmentation, due to the diversity of the medical imaging principle, the medical image processing may be easily disturbed by the following factors such as individual differences in imaging, bias field, motion artifacts, the complexity of the region of interest (ROI), the disease factor, the fuzzy boundary of ROI, and so on. Therefore, investigations on the medical image segmentation are always challenging and hot $[2,3]$. Currently, with the development of computer vision, superpixel-based segmentation algorithm is increasingly used in the field of medical image processing by researchers [4]. The simple linear iterative clustering (SLIC) is a gradient-ascent-based superpixel algorithm proposed by Achanta et al. in 2012 [5]. It has gradually attracted reseachers' attention and has been initially used in medical image processing

\footnotetext{
${ }^{*}$ Corresponding author: Benzheng Wei, College of Science and Technology, Shandong University of Traditional Chinese Medicine, Jinan 250355, China. Tel.: +86-53189628103; Fax: +86-53189628103; E-mail: wbz99@sina.com.
} 
because of the fast computational speed and excellent boundary adherence characteristic, etc. Jun Cheng and Jiang Liu et al. utilized SLIC to generate superpixels in retinal fundus images, then extracted features and segmented optic disc and optic cup on superpixel-level [6]. Shuangling Wang applied it in the feature extraction and segmentation of the neuronal electron microscopy images [7]. And in [8], it was also applied in the segmentation and edge detection of Crohn's disease in abdominal Magnetic Resonance Imaging (MRI) images by Dwarikanath Mahapatr et al.. However, these studies do not involve in the performance evaluation of SLIC on medical image segmentation.

To provide a better way to understand the performance of the algorithm, support the technical application and expand its application, its applicability and performance in medical image applications need to be verified. As medical image segmentation refers more to segmenting ROI and the boundaries of ROI are usually very blurry, this paper mainly focuses on the systematical analyses for the performance of SLIC from the indicators of computational speed, boundary recall and boundary accuracy, and superpixel uniformity.

The rest of the paper is organized as follows: Section 2 summarizes the advantage of SLIC on medical image processing and shortly introduces the SLIC algorithm; Section 3 presents an exhaustive analysis and the experimental results, and then the conclusion is drawn in the final section.

\section{SLIC algorithm}

Different medical imaging technology can obtain different types of medical images, such as CT images, MRI, ultrasound images. Bias field and motion artifacts often lead to the low unwrapping quality. The boundary of ROI is fuzzy. The SLIC algorithm is a superpixel-based segmentation algorithm, superior to pixel-level algorithm in terms of feature extraction and quantitative analysis, boundary information preservation and local information acquirement. Therefore, the SLIC algorithm has been applied to the medical image segmentation recently.

SLIC algorithm is an over-segmentation method, which selects cluster centers $C_{i}$ from the sampled regular grid spaced $\mathrm{S}$ to efficiently generate superpixels. $\mathrm{S}$ can be described as the following Eq. (1):

$$
S=\sqrt{N / K}
$$

where $\mathrm{N}$ is the number of the image pixels and $\mathrm{K}$ is the desired and settled number of the superpixels. Meanwhile, SLIC algorithm only searches for similar pixels from each cluster center to pixels for clustering within a $2 \mathrm{~S} * 2 \mathrm{~S}$ setting region instead of in the entire image, so it can generate superpixels faster.

SLIC combines the spatial distances with the color proximity in a distance measure D.

$$
D=\sqrt{\left[\frac{d_{c}}{N_{c}}\right]^{2}+\left[\frac{d_{s}}{N_{s}}\right]^{2}}
$$


where $d_{c}$ is color proximity and $d_{s}$ is space proximity, which can be described as $d_{c}=\sqrt{\left(l_{j}-l_{i}\right)^{2}+\left(a_{j}-a_{i}\right)^{2}+\left(b_{j}-b_{i}\right)^{2}}$ and $d_{s}=\sqrt{\left(x_{j}-x_{i}\right)^{2}+\left(y_{j}-y_{i}\right)^{2}} \quad$ respectively, where $[l, a, b]^{T}$ is the pixel's color representation in the CIELAB color space, and $[x, y]^{T}$ refers to the pixel's position.

\section{Experiment and analysis for the performance of SLIC on medical image processing}

In this paper, three indicators are introduced to analyze the applicability and performance of SLIC in medical image applications compared with Normalized cuts [9] and Turbopixels [10] algorithm, in terms of computational speed, boundary recall and boundary accuracy and superpixel uniformity. The datasets used in this paper contain 280 medical images, including 60 lung CT images, 60 cell microscopic images, 100 breast ultrasound images and 60 brain Magnetic Resonance (MR) images. The programs are implemented with Matlab (R2010b), and the experiments have been executed on Inter $2.93 \mathrm{G}$ dual-core processor with $3.29 \mathrm{G} \mathrm{RAM}$.

\subsection{Computational speed}

The computational speed of medical image segmentation algorithm can directly affect the efficiency of medical image processing, clinical application and researching application, so the computational speed experiments are the fundamental experiments to evaluate algorithm's performance.

\subsubsection{The impact of computational complexity}

The computational complexity of SLIC and Turbopixels algorithms is $O(N)$, while that of Normalized cuts is $O\left(N^{3 / 2}\right)$ [5,10]. The computational speeds are tested by segmenting 280 medical images, and the image sizes are settled as $128 * 128,256 * 256,512 * 512,1024 * 1024$ and $2048 * 2048$ separately(There are $280 * 5=1400$ images in total). The setting superpixel number is 300 . From the

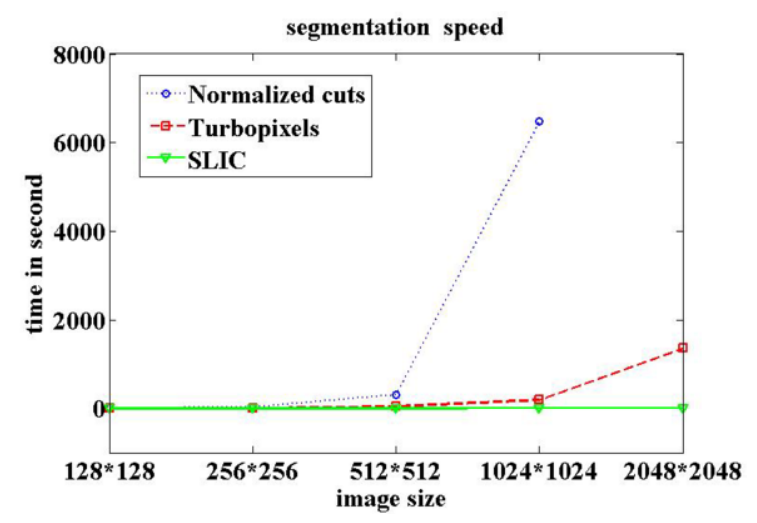

(a) The computational speed of SLIC, Turbopixels and Normalized cuts algorithms

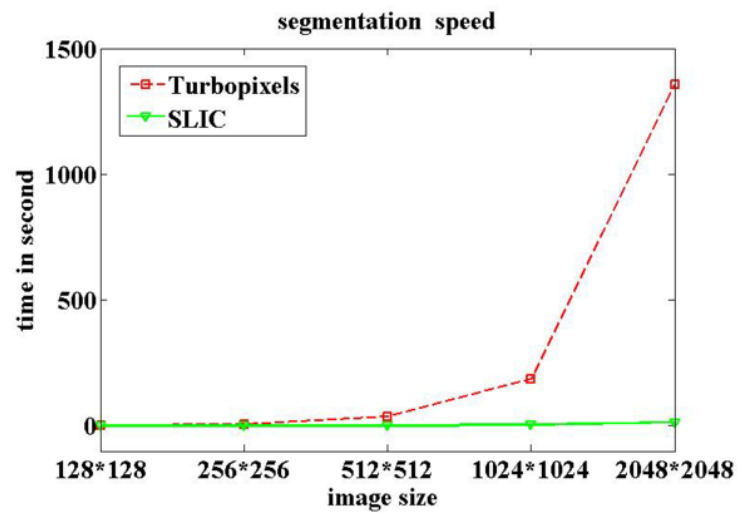

(b) The computational speed of SLIC and Turbopixels algorithms

Fig. 1. The computational speed of different algorithms. 
experimental results shown in Figure 1, it can be drawn that the SLIC algorithm computes faster than the Turbopixels algorithm. And with the increase of the image size, the advantages of SLIC become increasingly evident. Moreover, Normalized cuts algorithms usually get "out of memory" errors and fail to achieve the segmentation result during the image segmenting with the size of $2048 * 2048$.

\subsubsection{The sensitivity of the computational speed to image type}

There are some different types of medical images because of the complexity of the medical image sources. So it needs to test the sensitivity of the computational speed to image type, and the segmentation experiment contains 240 images, including 60 lung CT images, 60 cell microscopic images, 60 breast ultrasound images and 60 MR images. The number of superpixels is settled as 300 and the image size is $512 * 512$. The time consuming of the three methods is shown in Figure 2. As can be seen, for different types of images, the computational speed of SLIC varies within a smaller range, while Turbopixels and Normalized cuts algorithms have a greater change tendency. So, the computational speed of the SLIC algorithm is less sensitive to the image type.

\subsubsection{The sensitivity of the computational speed to the setting superpixel number}

The characteristic of ROI varies a lot under the influence of the disease factor and individual differences in imaging, the different size of superpixels have a great influence on the feature extraction. In this paper, the sensitivity of the computational speed to the setting superpixel number is tested by segmenting 240 images, and the setting superpixel number is separately settled as $200,300,400,500,600,700,800$ and 900 , with the image size of $512 * 512$. Figure 3 is the comparison's results for the time consuming of three methods. With the gradually increasing of the setting superpixel number, the time consuming of Normalized cuts algorithm increases sharply, while the Turbopixels and SLIC algorithms only have a little change. So, the computational speed of the SLIC algorithm is less sensitive to the setting superpixel number.

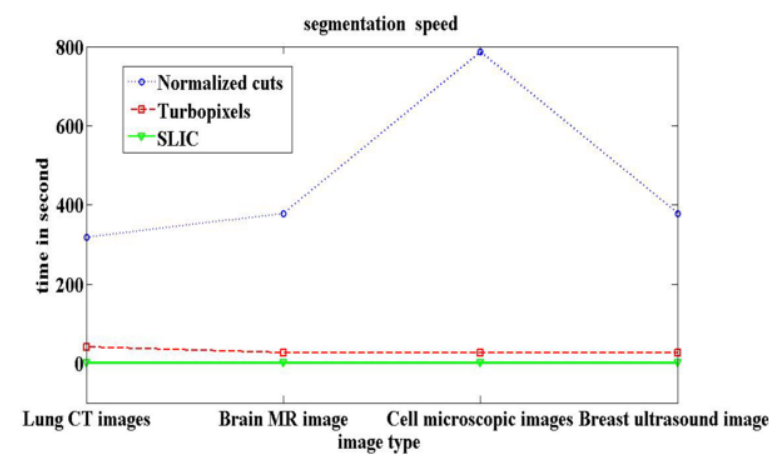

(a) The sensitivity of the computational speed for different image types of SLIC, Turbopixels and Normalized cuts algorithms

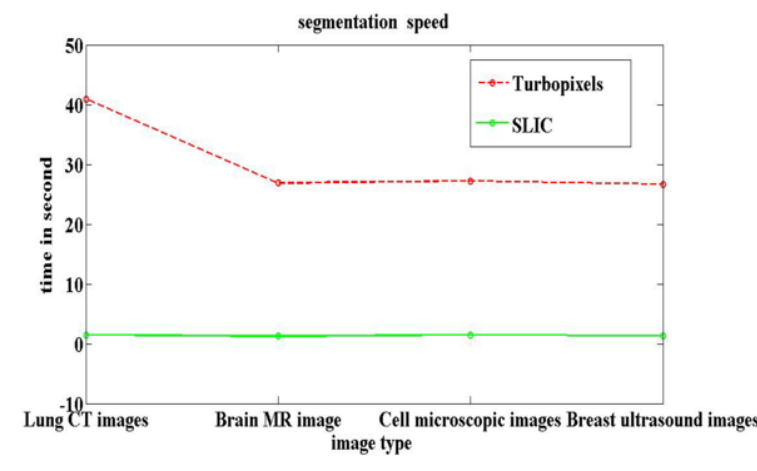

(b) The sensitivity of the computational speed to image type of SLIC and Turbopixels algorithms

Fig. 2. The sensitivity of the computational speed for different image types. 


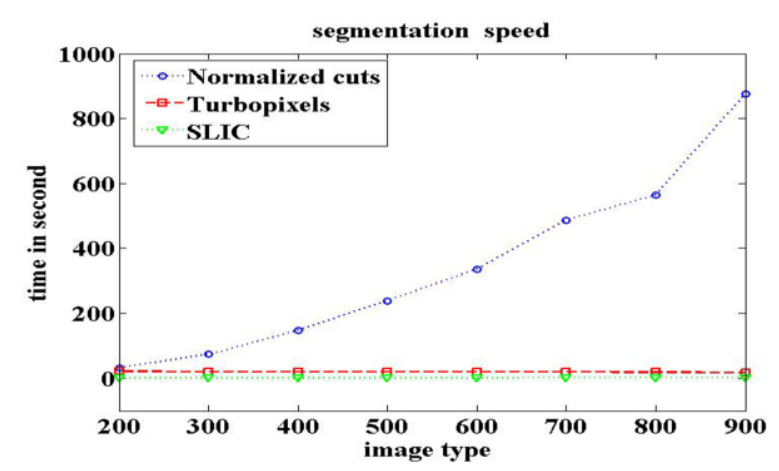

(a) The sensitivity of the computational speed to the setting superpixel number of SLIC, Turbopixels and Normalized cuts algorithms

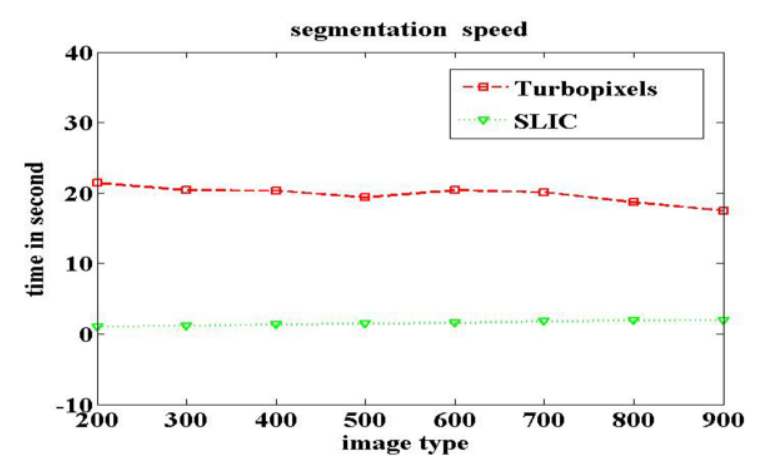

(b) The sensitivity of the computational speed to the setting superpixel number of SLIC and Turbopixels algorithms

Fig. 3. The sensitivity of the computational speed to the setting superpixel number.

The above experimental results show that the SLIC algorithm is faster, and it can be less sensitive to the image type and the setting superpixel number than the Turbopixels and Normalized cuts algorithms. Different degrees of sensitivity indicate different degrees of universality. So SLIC algorithm with the best universality can better meet the needs of medical image processing.

\subsection{Boundary recall and boundary accuracy}

The fuzzy characteristic of the boundary is a great drawback in the medical image segmentation [11]. To better characterize the accuracy of the image segmentation algorithms, the indicator of superpixel boundary on the boundary recall is employed in this paper according to [10,12]. In addition, the new indicator of the boundary accuracy is also designed and presented to test the robustness of fuzzy boundary.

The indicator of the boundary recall can measure what fraction of the edges of ROI fall a small distance threshold (16 neighborhoods in the experiments) of a superpixel boundary [5]. A high value of the boundary recall means that most of the real edges are obtained [5].

The indicator of boundary accuracy is utilized to measure what fraction of the edges of ROI covers the superpixel boundary. Difference from the boundary recall, the indicator of the boundary accuracy reflects the robustness of fuzzy boundary. The evaluation parameter BR is denoted as the indicator of boundary accuracy in this paper, and the equation is as follows,

$$
B R=\frac{N U M(A \cap B)}{B U M(B)}
$$

where $\mathrm{A}$ is the edges of ROI, B is the superpixel boundary, and $N U M()$ is the number of pixels of the region's boundaries. A high BR indicates that the algorithm is with high accuracy and better robustness of fuzzy boundary.

To evaluate the performance of these algorithms on superpixel boundary, 100 breast ultrasound images are separately segmented by these three algorithms with the image size of $512 * 512$ and the setting superpixel number of 500. Table 1 shows the performance of these three algorithms on the 
indicators of boundary recall and boundary accuracy. The experimental results show that SLIC has the best performance on indicators of boundary recall and boundary accuracy compared with Turbopixels and Normalized cuts algorithms, indicating that SLIC has the great robustness of fuzzy boundary.

\subsection{The superpixel uniformity}

Superpixel uniformity refers to that the superpixel's size is relatively fixed. Fixed-size superpixel can be of great benefit to local feature extraction and quantitative analysis of the lesions. So the superpixel uniformity can evaluate the performance of superpixel algorithm to a certain extent. The evaluation parameters Jvar is denoted as the superpixel uniformity in this paper. The formula of superpixel uniformity is designed as:

$$
J \operatorname{var}=\operatorname{var}(P)
$$

where $P=\left[Q_{1}, Q_{2}, Q_{3}, \ldots, Q_{i}\right]$, and $Q_{i}$ is the number of the ith superpixels, $\operatorname{var}(P)$ is the variance of P. The smaller Jvar is, the better superpixel uniformity will be.

To evaluate the superpixel uniformity of these three superpixel algorithms, 240 images with the size of 512*512 are segmented, including 60 lung CT image, 60 cell microscopic images, 60 breast ultrasound images and $60 \mathrm{MR}$ images with the setting superpixel number of 300 . As shown in Table 2, the Jvar of SLIC is the lowest, so SLIC has the best performance on superpixel uniformity.

The image segmentation results are shown in Figure 4 segmented by Turbopixels, Normalized cuts and SLIC algorithms. For the breast ultrasound image, SLIC algorithm has the better boundary, the fixed size and shape of superpixels, while Turbopixels and Normalized cuts algorithms have the nonuniformity superpixels as shown in Figure 4.

Table 1

The performance of the three algorithms on boundary recall and boundary accuracy

\begin{tabular}{|l|l|l|l|}
\hline Algorithm & Normalized cuts & Turbopixels & SLIC \\
\hline Boundary recall & 0.4175 & 0.6275 & 0.8258 \\
\hline Boundary accuracy & 0.0623 & 0.0893 & 0.2935 \\
\hline
\end{tabular}

Table 2

Superpixel uniformity of the different algorithms

\begin{tabular}{|l|l|l|l|}
\hline & Normalized cuts & Turbopixels & SLIC \\
\hline Lung CT image & 0.0664 & 0.0385 & 0.0243 \\
\hline Cell microscopic image & 0.0684 & 0.0386 & 0.0204 \\
\hline Breast ultrasound image & 0.0675 & 0.0358 & 0.0258 \\
\hline Brain MR images & 0.0641 & 0.0307 & 0.0246 \\
\hline The mean in different types images & 0.0666 & 0.0359 & 0.0238 \\
\hline
\end{tabular}




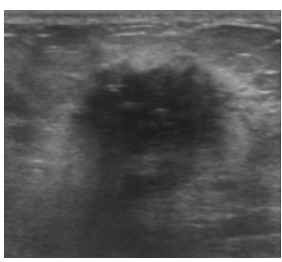

(a) Original images

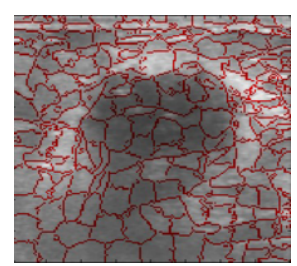

(b) The segmentation results by Normalized cuts

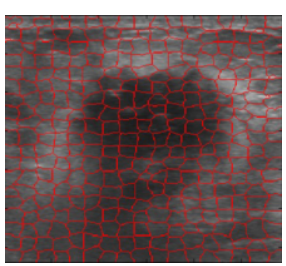

(c) The segmentation results by Turbopixels

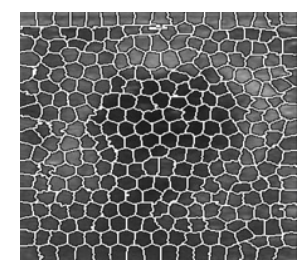

(d) The segmentation results by SLIC

Fig. 4. The segmentation results of breast ultrasound images segmented by Normalized cuts, Turbopixels and SLIC algorithms.

By getting the synthetically estimated value of those indicators and comparing with Turbopixels and Normalized cuts algorithms, SLIC algorithm is faster and can be less sensitive to the image type and the setting superpixel number, so it has great universality. Because of its high boundary recall and excellent robustness of fuzzy boundary, the fixed-size of superpixels and the accurate result, SLIC algorithm can also have great advantages in local feature extraction and quantitative analysis of the lesions ROI. Therefore, from the experiment results, it can be drawn that SLIC algorithm suits well for medical image segmentation with the characteristic of complicated sources, fuzzy boundary and so on.

\section{Conclusion}

In this paper, two indicators of boundary accuracy and superpixel uniformity are introduced with other indicators to achieve the systematical analysis on the medical image segmentation performance of SLIC algorithm. A high boundary accuracy value indicates that the algorithm has better robustness of fuzzy boundary. Fixed-size superpixels are beneficial to local feature extraction and quantitative analysis of the lesions ROI. Therefore, superpixel uniformity is one important indicator to select a segmentation algorithm. With fast computational speed, high boundary recall, excellent robustness of fuzzy boundary, the fixed-size of superpixels and accurate segmentation performance, SLIC algorithm can do well in medical image segmentation. Due to its good performance, the medical image segmentation based on SLIC algorithm should be one of the deserved research domain in the future.

\section{Acknowledgment}

The work is partly support by the NSFC Joint Fund with Guangdong under Key Project (No. U1201258), Natural Science Foundation of Shandong Province (No. ZR2011FQ033), Institute Support Innovation Project of Jinan Administration of Science \& Technology (No. 201202012, 201303004), Jinan Youth Science and Technology Star Project (No. 20110302). 


\section{References}

[1] B. Peng, L. Zhang and D, Zhang, A survey of graph theoretical approaches to image segmentation, Pattern Recognition 46 (2013), 1020-1038.

[2] D.L. Pham, C.Y. Xu and J.L. Prince, Current methods in medical image segmentation 1, Annual Review of Biomedical Engineering 2 (2000), 315-337.

[3] Q. Wang, X.L. Song and Z.G. Jiang, An improved image segmentation method using three-dimensional region growing algorithm, International Conference on Information Science and Computer Applications 92 (2013), 148-152.

[4] A. Lucchi, K. Smith, R. Achanta, V. Lepetit and P. Fua, A fully automated approach to segmentation of irregularly shaped cellular structures in EM images, Medical Image Computing and Computer-Assisted Intervention 6362 (2010) 463-471.

[5] R. Achanta, A. Shaji, K. Smith, A. Lucchi, P. Fua, and S. Susstrunk, SLIC superpixels compared to state-of-the-art superpixel methods, IEEE Transactions on Pattern Analysis and Machine Intelligence 34 (2012), 2274-2282.

[6] J. Cheng, J. Liu, Y. Xu, F. Yin, D.W. Wong, N.M. Tan, D. Tao, C.Y. Cheng, T. Aung and T.Y. Wong. Superpixel classification based optic disc and optic cup segmentation for glaucoma screening, IEEE Transactions on Medical Imaging 32 (2013), 1019-1032.

[7] S.L. Wang, G.B. Cao, B.Z. Wei, Y.L. Yin, G.P. Yang and C.M. Li, Hierarchical level features based trainable segmentation for electron microscopy images, Biomedical Engineering Online 12 (2013), 59.

[8] D. Mahapatra, P.J. Schüffler, J.A.W. Tielbeek, J. Makanyanga, J. Stoker, S. Taylor, F. Vos and J. Buhmann, Automatic detection and segmentation of Crohn's disease tissues from abdominal MRI, IEEE Transactions on Medical Imaging 32 (2013), 2332-2347.

[9] J. Shi and J. Malik, Normalized cuts and image segmentation, IEEE Trans. Pattern Analysis and Machine Intelligence 22 (2000), 888-905.

[10] A. Levinshtein, A. Stere, K. Kutulakos, D. Fleet, S. Dickinson and K. Siddiqi, Turbopixels: fast superpixels using geometric flows, IEEE Trans. Pattern Analysis and Machine Intelligence 31 (2009), 2290-2297.

[11] X. Fan, J. Yang, Y. Zheng, L. Cheng and Y. Zhu, A novel unsupervised segmentation method for MR brain images based on fuzzy methods, Computer Vision for Biomedical Image Applications 3765 (2005), 160-169.

[12] O. Veksler, Y. Boykov and P. Mehrani, Superpixels and supervoxels in an energy optimization framework, Proc. European Conf. Computer Vision 6315 (2010), 211-214. 